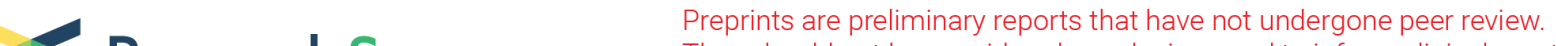 $\begin{array}{ll}\text { Research Square } & \text { They should not be considered conclusive, used to inform clinical practice, } \\ \text { or referenced by the media as validated information. }\end{array}$
}

\section{Risk Factors for Postoperative lleus after Diverting Loop Ileostomy Closure following Rectal Cancer Surgery: A Retrospective Study}

Toshihiro Nakao ( $\square$ nakao.toshihiro@tokushima-u.ac.jp )

Tokushima University Hospital: Tokushima Daigaku Byoin https://orcid.org/0000-0002-2827-0315

Mitsuo Shimada

Tokushima University Hospital: Tokushima Daigaku Byoin

Kozo Yoshikawa

Tokushima University Hospital: Tokushima Daigaku Byoin

Takuya Tokunaga

Tokushima University Hospital: Tokushima Daigaku Byoin

Masaaki Nishi

Tokushima University Hospital: Tokushima Daigaku Byoin

Hideya Kashihara

Tokushima University Hospital: Tokushima Daigaku Byoin

Chie Takasu

Tokushima University Hospital: Tokushima Daigaku Byoin

Yuma Wada

Tokushima University Hospital: Tokushima Daigaku Byoin

Toshiaki Yoshimoto

Tokushima University Hospital: Tokushima Daigaku Byoin

Syoko Yamashita

Tokushima University Hospital: Tokushima Daigaku Byoin

Yosuke Iwakawa

Tokushima University Hospital: Tokushima Daigaku Byoin

\section{Research Article}

Keywords: Postoperative ileus, lleostomy closure, Diverting loop ileostomy, Risk factor, Rectal cancer

Posted Date: August 23rd, 2021

DOI: https://doi.org/10.21203/rs.3.rs-812322/v1 
License: (c) (i) This work is licensed under a Creative Commons Attribution 4.0 International License. Read Full License 


\section{Abstract}

Background: Postoperative ileus is one of the most common complications after diverting loop ileostomy closure. Some reports have investigated the risk factors for postoperative complications or ileus after ileostomy closure; however, these studies did not evaluate the index surgery sufficiently. In this study, we evaluated the risk factors, including the details of the index surgery, for ileus after diverting ileostomy closure.

Methods: This was a retrospective study of patients who underwent ileostomy closure following index surgery for rectal cancer. Patients who developed postoperative ileus (POI (+)) and patients who did not (POI (-)) after ileostomy closure were compared.

Results: Fifty-nine patients were evaluated and were divided into two groups: $\mathrm{POI}(+)(\mathrm{n}=9)$ and POI (-) $(n=50)$, and the groups were compared. There were no significant differences in the details of the index surgery, operative procedure, transanal total mesorectal excision, lateral lymph node dissection, operating time, or blood loss. The incidence of Clavien-Dindo grade $\geq \mathrm{III}$ complications after the index surgery was significantly higher in the POI (+) group.

Conclusions: The incidence of Clavien-Dindo grade $\geq$ III complications after the index surgery may increase the risk of postoperative ileus after ileostomy closure.

\section{Background}

Anastomotic leakage is one of the most serious complications after rectal cancer surgery. Various methods have been used to prevent anastomotic leakage, such as combined mechanical and oral antibiotic bowel preparation, transanal tube placement, and evaluating the anastomosis with the indocyanine green (ICG) fluorescence method[1-3]. Diverting loop ileostomy is often performed for cases with a higher risk of developing anastomotic leakage[4]. Newer surgical techniques and devices have been developed, such as transanal total mesorectal excision (TaTME), that make it possible to anastomose for rectal cancer near the anus, and these techniques and developments have increased the indications for diverting loop ileostomy to prevent anastomotic leakage. Generally, the ileostomy is closed a few weeks or months after the surgery. Regardless of the fact that ileostomy closure is a relatively easy surgery, several postoperative complications often arise[5]. In particular, postoperative ileus is one of the most common complications.

We have performed diverting loop ileostomy in rectal cancer surgery for patients with a higher risk of developing anastomotic leakage. The risk factors for anastomotic leakage are male sex, high body mass index (BMI), high American Society of Anesthesiologists physical status (ASA-PS), large tumor size, preoperative chemotherapy, low-level anastomosis, multiple stapler firings, long operative time, high intraoperative transfusion/blood loss, and lack of a pelvic drain. We usually close the ileostomy from three to six months after the surgery [6]. 
Some reports have investigated the risk factors for postoperative complications or ileus after ileostomy closure[7-10]. However, these studies did not focus on the details of the index surgery, such as TaTME and lateral lymph node dissection (LLND). In this study, we evaluated the risk factors, including the details of the index surgery, for ileus after diverting ileostomy closure.

\section{Methods}

\section{Patients}

This was a retrospective study of patients who underwent ileostomy closure in Tokushima University Hospital between January 2017 and April 2021. The protocol was approved by the Ethics Committee of Tokushima University (approval number. 3215-1), and the study was conducted in accordance with the provisions of the Declaration of Helsinki. All patients provided informed consent for the use of their data. Diverting loop ileostomy was performed when the patients underwent surgery for rectal cancer, gastrointestinal stromal tumor, or neuroendocrine tumor. Anterior resection (AR), intersphincteric resection (ISR), total colectomy (TC), or transanal minimally invasive surgery (TAMIS) was performed for rectal tumors, and all surgeries were performed laparoscopically. Performing TaTME and LLND depended on the patient and tumor progression. Some of the patients diagnosed with stage II-IV lower rectal cancer underwent adjuvant radiotherapy, and some diagnosed with stage III or IV rectal cancer underwent adjuvant chemotherapy or neoadjuvant chemotherapy, respectively, depending on the patient's condition. Patients were divided into two groups: a POI (+) group, which represented patients who developed postoperative ileus after ileostomy, and a $\mathrm{POI}(-)$ group, which represented patients who did not develop postoperative ileus.

\section{lleostomy Closure}

The ileostomy was closed between 1 and 25 months postoperatively. Ileostomies in patients receiving adjuvant chemotherapy were closed after completing adjuvant chemotherapy. All patients underwent water-soluble contrast enema and colonoscopy to check the patency and integrity of the anastomosis before ileostomy closure. Food intake was stopped, and mechanical bowel preparation was performed the day before surgery. Our standard operative technique for ileostomy closure began with a peristomal skin incision, then the proximal and distal limbs were dissected to the peritoneal cavity, and the loop was resected, followed by anastomosis. Anastomosis was hand-sewn end-to-end or side-to-side. The peritoneum and fascia were closed with interrupted sutures, and the skin was closed with a purse string closure or linear closure. The anastomosis and skin closure methods depended on the surgeon's preference. Antimicrobial prophylaxis was used from just before surgery until the first day after surgery, and food intake was started from the third day after surgery. Patients were discharged on the eighth day or later after surgery, if there were no problems. 


\section{Definition Of lleus}

lleus was defined as digestive symptoms, such as abdominal pain and distension, with nausea and vomiting, and X-ray and computed tomography (CT) findings, such as intestinal dilation and multiple airfluid levels throughout the abdomen.

\section{Statistical Analysis}

The collected patient data were reviewed. All statical analyses were performed with EZR (Version 1.54) (Saitama Medical Center, Jichi Medical University, Saitama, Japan), a graphical user interface for R (version 4.03) (R Foundation for Statical Computing, Vienna, Austria)[11]. More precisely, EZR is a modified version of $\mathrm{R}$ commander (version 2.7-1) designed to add statistical functions used frequently in biostatistics. Categorical variables were analyzed with Fisher's exact test, and continuous variables were analyzed with Student's $t$ test. A $p$ value of less than 0.05 was considered statistically significant.

\section{Results}

The patients' characteristics are summarized in Table 1. A total 59 patients, 42 (71.2\%) male and 17 (28.8\%) female, underwent closed ileostomy between January 2017 and April 2021. The mean age was 64.8 years, and the mean body mass index was $22.6 \mathrm{~kg} / \mathrm{m}^{2}$. Twenty-one (35.6\%), 31 (52.5\%), and 7 $(11.9 \%)$ patients had ASA-PS I, II, and III, respectively. The primary diseases were rectal cancer (stage 0: 1 (1.7\%), I: 21 (35.6\%), II: 16 (27.1\%), III: 13 (22.0\%), IV: 5 (8.5\%), and unknown: 1 (1.7\%)), gastrointestinal stromal tumor (1 (1.7\%)), and neuroendocrine tumor (1 (1.7\%). Laparoscopic AR, ISR, TC, and TAMIS were performed for $41(69.5 \%), 16(27.1 \%), 1$ (1.7\%), and 1 (1.7\%) patients, respectively. Among the procedures, TaTME was performed for 28 (47.5\%) patients, and LLND was performed for 12 (20.3\%) patients. The mean operating time for the index surgery was $350.6 \mathrm{~min}$, and mean blood loss was $61.7 \mathrm{ml}$. Complications (Clavien-Dindo grade $\geq \mathrm{III}$ ) developed in 5 (8.5\%) patients after the index surgery. Complications comprised two grade Illa ileus, two grade IIla anastomotic stenosis, and 1 grade IIIb neurogenic bladder. Three (5.1\%) patients underwent neoadjuvant radiotherapy, and $13(22.0 \%)$ patients underwent neoadjuvant chemotherapy; 18 (30.5\%) patients underwent adjuvant chemotherapy. The interval between the index surgery and ileostomy closure was 181.3 days. The ileostomy was resected and anastomosed. End-to-end anastomosis was performed for 53 (89.8\%) patients, and side-to-side anastomosis was performed for $6(10.2 \%)$ patients. The skin was closed with a purse string closure in 53 $(89.8 \%)$ patients and with a linear closure in $6(10.2 \%)$ patients. The mean operating time was 92.9 min, and the mean blood loss was $10.4 \mathrm{ml}$. Nine (15.3\%) patients developed ileus after surgery; no patients developed anastomotic leakage or wound infection. Food intake was started a mean of 6.5 days after surgery, and the mean hospital stay after surgery was 11.6 days.

The POI (+) group and the POI (-) group were compared (Table 2). There were nine patients in the POI (+) group and 50 patients in the $\mathrm{POI}(-)$ group. There was no significant difference in age, sex, ASA-PS, BMI, or cancer stage between the groups. There was a significant difference in operative procedure for the 
index surgery ( $p=0.037)$; however, there was no difference regarding TaTME or LLND. There was also no difference in operating time and blood loss for the index surgery between the groups. In the POI (+) group, the incidence of Clavien-Dindo grade $\geq \mathrm{III}$ complications after the index surgery was significantly higher than in the $\mathrm{POI}(-)$ group $(p=0.022)$. There was no difference regarding neoadjuvant radiotherapy or neoadjuvant chemotherapy for the primary disease. However, adjuvant chemotherapy was administered significantly more often in the POI (-) group compared with the POI $(+)$ group $(p=0.046)$. The interval between the index surgery and ileostomy closure did not differ between the groups. Regarding the ileostomy closure technique, there was no significant difference between end-to-end anastomosis and side-to-side anastomosis, and there was no significant difference between purse string skin closure and linear skin closure between the groups. Regarding the surgical outcomes, operating time and blood loss did not differ significantly between the groups. Food intake was later, and hospital stay was longer, in the $\mathrm{POI}(+)$ group than in the POI (-) group.

\section{Discussion}

lleus is one of the most common postoperative complications after ileostomy closure, with a reported incidence of $16.4-33.0 \%$. Postoperative ileus developed in $4.9-16.8 \%$ patients after ileostomy closure[7, $9,10]$. In this study, the incidence of postoperative complications after ileostomy closure was $22.6 \%$, and the incidence of ileus was $14.5 \%$.

Some repots have investigated the risk factors for postoperative complications or ileus after ileostomy closure[7-10]. However, these studies did not focus on the details of the index surgery, and the relationship between the index surgery and postoperative ileus after ileostomy closure has not been sufficiently evaluated. Laparotomy and total colectomy during the index surgery are reported risk factors for postoperative ileus after ileostomy[8]. In the present study, all index surgeries were performed laparoscopically, and there was a significant difference in laparoscopic operative procedures between the $\mathrm{POI}(+)$ and $\mathrm{POI}(-)$ groups. TC was performed in only the POI (+) group. There was no significant difference in TaTME, LLND, operating time, and blood loss between the groups. Clavien-Dindo grade $\geq$ III complications were significantly more common in the POI (+) group. Additionally, grade Illa ileus, grade IIla anastomotic stenosis, and grade IIIb neurogenic bladder occurred in the POI (+) group; the patient who developed neurogenic bladder underwent cystostomy. Previous studies reported the risk factors for postoperative ileus as low albumin, opioid use, long duration of surgery, emergency surgery, and blood loss requiring transfusion[12]. Invasive treatment for complications following the index surgery might also be related to the incidence of postoperative ileus after ileostomy closure.

Previous studies also reported that neoadjuvant radiotherapy, neoadjuvant chemotherapy, and adjuvant chemotherapy did not affect the incidence of postoperative ileus after ileostomy closure[9]. In the present study, there was no difference in neoadjuvant radiotherapy and chemotherapy administration between the $\mathrm{POI}(+)$ and $\mathrm{POI}(-)$ groups. However, significantly more patients received adjuvant chemotherapy in the POI (-) group than in the POI (+) group. The indication for adjuvant chemotherapy was stage III or IV rectal cancer, and there was no difference in the rate of adjuvant chemotherapy between patients with 
stage III vs IV rectal cancer. Cancer progression because of not undergoing adjuvant chemotherapy might contribute to the development of postoperative ileus.

This study suggested that there was no relationship between the interval between the index surgery and ileostomy closure and postoperative ileus after ileostomy closure[13]. Previous study also reported that postoperative complication was similar in early ileostomy closure group and late closure group[14].

Regarding the ileostomy closure technique, there was no significant difference in anastomotic leakage rates between hand-sewn and stapled techniques in one study, but the rate of small bowel obstruction and ileus were significantly lower with the stapled technique[15]. In the present study, $88.7 \%$ of the patients underwent end-to-end anastomosis, and $11.3 \%$ patients underwent side-to-side anastomosis; all but one of the anastomoses were hand-sewn. There was no significant difference in the rate of ileus between end-to-end and side-to-side anastomosis, and between the hand-sewn and stapled techniques.

The limitations of this study are the retrospective, single-center design, and the small sample seize.

\section{Conclusions}

Postoperative ileus is a common complication after diverting loop ileostomy closure. Complications after the index surgery may increase the risk of postoperative ileus after ileostomy closure.

\section{List Of Abbreviations}

AR Anterior resection

ASA-PS American Society of Anesthesiologists physical status

BMI body mass index

CT computed tomography

ICG indocyanine green

ISR intersphincteric resection

LLND lateral lymph node dissection

TAMIS transanal minimally invasive surgery

TaTME transanal total mesorectal excision

TC total colectomy

\section{Declarations}




\section{Ethics approval and consent to participate}

This study was approved by the Ethics Committee of Tokushima University (approval number. 3215-1), and the study was conducted in accordance with the provisions of the Declaration of Helsinki. All patients provided informed consent for the use of their data.

\section{Consent for publication}

Not applicable

\section{Availability of data and materials}

The datasets used and/or analysed during the current study are available from the corresponding author on reasonable request.

\section{Competing interests}

The authors declare that they have no competing interests.

\section{Funding}

The authors declare that they have no financial interests.

\section{Authors' contributions}

TN, MS and KY were involved in study design and data interpretation. TT, MN, HK, CT, YW, SY and YI were involved in the data analysis. All authors revised the manuscript, approved the manuscript to be published, and agree to be accountable for all aspects of the work in ensuring that questions related to the accuracy or integrity of any part of the work are appropriately investigated and resolved.

\section{Acknowledgements}

We thank Jane Charbonneau, DVM, from Edanz (https://jp.edanz.com/ac) for editing a draft of this manuscript.

\section{References}

1. Scarborough JE, Mantyh CR, Sun Z, Migaly J. Combined mechanical and oral antibiotic bowel preparation reduces incisional surgical site infection and anastomotic leak rates after elective colorectal resection: An analysis of colectomy-targeted ACS NSQIP. Annals of Surgery. 262: Lippincott Williams and Wilkins; 2015. pp. 331-7. 
2. Hidaka E, Ishida F, Mukai S, Nakahara K, Takayanagi D, Maeda C, et al. Efficacy of transanal tube for prevention of anastomotic leakage following laparoscopic low anterior resection for rectal cancers: a retrospective cohort study in a single institution. Surgical Endoscopy. Springer Science and Business Media, LLC; 2015;29:863-7.

3. Shen R, Zhang Y, Wang T. Indocyanine green fluorescence angiography and the incidence of anastomotic leak after colorectal resection for colorectal cancer: A meta-analysis. Diseases of the Colon and Rectum. 61: Lippincott Williams and Wilkins; 2018. pp. 1228-34.

4. Defunctioning_Stoma_Reduces_Symptomatic.8.

5. Kaidar-Person O, Person B, Wexner SD. Complications of construction and closure of temporary loop ileostomy. Journal of the American College of Surgeons. 2005. p. 759-73.

6. Qu H, Liu Y, Bi D song. Clinical risk factors for anastomotic leakage after laparoscopic anterior resection for rectal cancer: a systematic review and meta-analysis. Surgical Endoscopy. Springer New York LLC; 2015;29:3608-17.

7. Garfinkle R, Filion KB, Bhatnagar S, Sigler G, Banks A, Letarte F, et al. Prediction model and webbased risk calculator for postoperative ileus after loop ileostomy closure. British Journal of Surgery. John Wiley and Sons Ltd; 2019;106:1676-84.

8. Aktaş A, Kayaalp C, Ateş M, Dirican A. Risk factors for postoperative ileus following loop ileostomy closure. Turkish Journal of Surgery Bilimsel Tip Yayinevi. 2020;36:333-9.

9. Man VCM, Choi HK, Law WL, Foo DCC. Morbidities after closure of ileostomy: analysis of risk factors. International Journal of Colorectal Disease Springer Verlag. 2016;31:51-7.

10. Mansfield SD, Jensen C, Phair AS, Kelly OT, Kelly SB. Complications of loop ileostomy closure: A retrospective cohort analysis of 123 patients. World J Surg. 2008;32:2101-6.

11. Kanda Y. Investigation of the freely available easy-to-use software "EZR" for medical statistics. Bone Marrow Transplant. 2013;48:452-8.

12. Bragg D, El-Sharkawy AM, Psaltis E, Maxwell-Armstrong CA, Lobo DN. Postoperative ileus: Recent developments in pathophysiology and management. Clinical Nutrition. Churchill Livingstone; 2015. pp. 367-76.

13. Wang L, Chen X, Liao C, Wu Q, Luo H, Yi F, et al. Early versus late closure of temporary ileostomy after rectal cancer surgery: a meta-analysis. Surgery Today. Springer; 2021. p. 463-71.

14. Farag S, Rehman S, Sains P, Baig MK, Sajid MS. Early vs delayed closure of loop defunctioning ileostomy in patients undergoing distal colorectal resections: an integrated systematic review and meta-analysis of published randomized controlled trials. Colorectal Disease. Blackwell Publishing Ltd; 2017. pp. 1050-7.

15. Madani R, Day N, Kumar L, Tilney HS, Gudgeon AM. Hand-Sewn versus Stapled Closure of Loop Ileostomy: A Meta-Analysis. Digestive Surgery. S. Karger AG; 2019. pp. 183-94.

\section{Tables}


Due to technical limitations, table 1 and 2 is only available as a download in the Supplemental Files section.

\section{Supplementary Files}

This is a list of supplementary files associated with this preprint. Click to download.

- Table1.xlsx

- Table2.xlsx 